\title{
ICTV Virus Taxonomy Profile: Retroviridae 2021
}

\author{
John Coffin', Jonas Blomberg ${ }^{2}$, Hung Fan ${ }^{3}$, Robert Gifford ${ }^{4}$, Theodora Hatziioannou ${ }^{5}$, Dirk Lindemann ${ }^{6}$, Jens Mayer ${ }^{7}$, \\ Jonathan Stoye ${ }^{8}$, Michael Tristem ${ }^{9}$, Welkin Johnson ${ }^{10, *}$ and ICTV Report Consortium
}

\begin{abstract}
Viruses in the family Retroviridae are found in a wide variety of vertebrate hosts. Enveloped virions are $80-100 \mathrm{~nm}$ in diameter with an inner core containing the viral genome and replicative enzymes. Core morphology is often characteristic for viruses within the same genus. Replication involves reverse transcription and integration into host cell DNA, resulting in a provirus. Integration into germline cells can result in a heritable provirus known as an endogenous retrovirus. This is a summary of the International Committee on Taxonomy of Viruses (ICTV) Report on the family Retroviridae, which is available at ictv.global/ report/retroviridae.
\end{abstract}

Table 1. Characteristics of members of the family Retroviridae

\begin{tabular}{|ll|}
\hline Example: & Moloney murine leukemia virus (AF033811), species Murine leukemia virus, genus Gammaretrovirus \\
\hline Virion & Enveloped spheres of $80-100 \mathrm{~nm}$ diameter with $8 \mathrm{~nm}$ glycoprotein spikes \\
Genome & Dimer of positive-sense, ssRNA $(7-13 \mathrm{~kb})$; may be partially reverse-transcribed in virions of spumaretroviruses \\
Replication & dsDNA produced by reverse transcription of the RNA genome is integrated into host genome and serves as template for transcription \\
Translation & From capped and polyadenylated genomic transcripts and subgenomic, spliced mR As \\
Host range & Rertebrates \\
Taxonomy & Spumaretrovirinae include $>10$ genera and $>65$ species
\end{tabular}

\section{VIRION}

Virions are spherical, enveloped and $80-100 \mathrm{~nm}$ in diameter (Table 1, Fig. 1) [1] with $8 \mathrm{~nm}$-long glycoprotein surface projections. The internal core constitutes the viral nucleocapsid. The apparently spherical nucleocapsid is rod or truncated cone-shaped for members of the genus Lentivirus, eccentric for members of the genus Betaretrovirus and concentric for members of the genera Alpharetrovirus, Gammaretrovirus, Deltaretrovirus and the subfamily Spumaretrovirinae.

\section{GENOME}

The genome of members of the subfamily Orthoretrovirinae is a dimer of linear positive-sense ssRNA of $7-13 \mathrm{~kb}$ [1]. The component monomers are linked by hydrogen bonds, and are polyadenylated at the $3^{\prime}$-end and possess a type 1 5 '-cap structure. Purified virion RNA is not infectious. Each monomer is associated with a specific tRNA molecule that is base-paired to the primer binding site near the $5^{\prime}$-end of the RNA. A proportion of virions of members of the Spumaretrovirinae may contain dsDNA (5-10\%), derived from reverse

Received 10 November 2021; Accepted 11 November 2021; Published 23 December 2021

Author affiliations: ${ }^{1}$ Tufts University, Boston, MA 2111, USA; ${ }^{2}$ Uppsala University, Sweden; ${ }^{3}$ University of California, Irvine, CA $92697-3905$, USA; ${ }^{4}$ Center for Virus Research, Glasgow G61 1QH, UK; ${ }^{5}$ The Rockefeller University, New York, NY10065, USA; ${ }^{6}$ Technische Universität Dresden, Dresden, 01307, Germany; ${ }^{7}$ University of Saarland, 66421 Homburg/Saar, Germany; ${ }^{8}$ Francis Crick Institute, 1 Midland Road, London NW1 1 AT, UK; ${ }^{9}$ Imperial College London, Berkshire, SL5 7PY, UK; ${ }^{10}$ Boston College, Chestnut Hill, MA 02467, USA.

*Correspondence: Welkin Johnson, welkin.johnson@bc.edu

Keywords: ICTV Report; taxonomy; Retroviridae; HIV; AIDS.

Abbreviations: LTR, long terminal repeat; SU, Env surface subunit; TM, Env transmembrane subunit.

†Deceased

001712 (c) 2021 
(a)

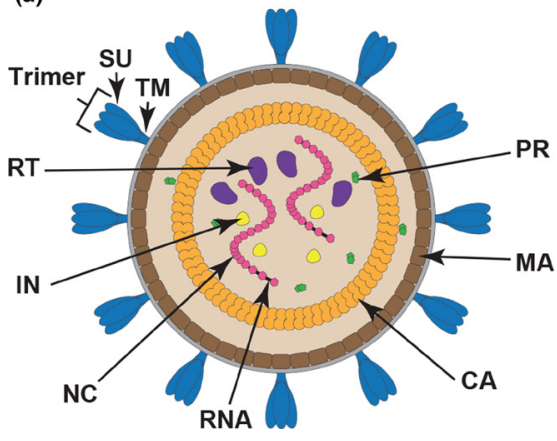

(b)

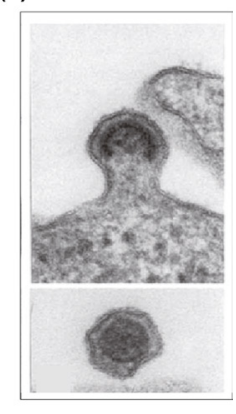

(a) murine leukemia virus (8.3 kb)

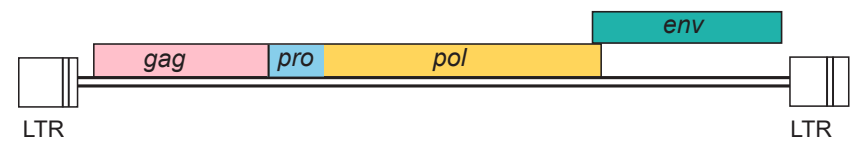

(b) simian foamy virus $(13.2 \mathrm{~kb})$

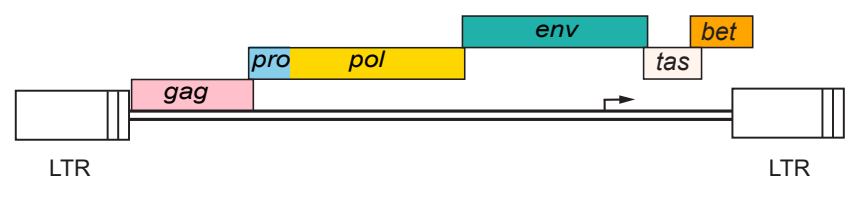

Fig. 1. (a) Schematic diagram of a retrovirus particle (courtesy of B. Lawhorn). SU, surface and TM, transmembrane subunits of the envelope protein; RT, reverse transcriptase; IN, integrase; NC, nucleocapsid; CA, capsid; MA, matrix; PR, protease. (b) Transmission electron micrograph of a murine leukemia virus particle. Credit M. Gonda, reproduced from [4], by permission of Cold Spring Harbor Laboratory Press.

transcription of viral genomic RNA genome during assembly and egress from the cell.

\section{REPLICATION}

The entry glycoprotein Env forms a heterotrimer comprising surface (SU) and transmembrane (TM) subunits. Binding is mediated by the SU subunit to specific receptors on the cell surface, resulting in fusion of the viral envelope with the plasma membrane $[2,3]$. Reverse transcription of the RNA genome produces dsDNA with long terminal repeats (LTRs) (Fig. 2) [4]. The dsDNA is integrated into the host cell genome to form the provirus [5], which serves as a template for synthesis of viral genomes and mRNAs by RNA polymerase II. Capsids assemble either at the plasma membrane (members of most genera) or as intracytoplasmic particles (for members of the subfamily Spumaretrovirinae and members of the genus Betaretrovirus). Virions are released from the cell by budding and undergo proteolytic maturation [6].

\section{PATHOGENICITY}

Many retroviruses are important human and veterinary pathogens, associated with a variety of diseases, including leukemias, lymphomas, sarcomas and other tumours of mesodermal origin; carcinomas of mammary tissue, liver, lung and kidney; immunodeficiencies (e.g. AIDS); autoimmune diseases; motor neuron diseases; and several acute diseases involving tissue damage $[4,7]$. Transmission is horizontal via a number of routes, including blood, saliva and sexual contact, and via direct infection of the developing embryo, or via milk or perinatal routes.

\section{TAXONOMY}

Current taxonomy: ictv.global/taxonomy. The family Retroviridae belongs to the order Ortervirales [8]. Currently, classification of subfamilies, genera and species is based primarily

Fig. 2. Genome organization of representatives of the subfamilies (a) Orthoretrovirinae and (b) Spumaretrovirinae. Arrowhead indicates an internal promoter found in the foamy virus genome.

on phylogenetic analysis, genome characteristics (ssRNA or ssRNA partially reverse-transcribed in the virion, size) and the presence or absence of specific regulatory and accessory genes.

\section{RESOURCES}

Full ICTV Report on the family Retroviridae: ictv.global/ report/retroviridae.

Funding information

Production of this summary, the online chapter, and associated resources was funded by a grant from the Wellcome Trust (WT108418AIA).

\section{Acknowledgements}

Members of the ICTV Report Consortium are Stuart G. Siddell, Elliot J. Lefkowitz, Sead Sabanadzovic, Peter Simmonds, F. Murilo Zerbini, Donald B. Smith, and Balázs Harrach.

Conflicts of interest

The authors declare that there are no conflicts of interest

References

1. Goff SP. Retroviridae. In: Knipe DM and Howley PM (eds). Fields Virology: Lippincott, Williams \& Wilkins; 2013. pp. 1424-1473.

2. Lindemann D, Steffen I, Pöhlmann S. Cellular entry of retroviruses. Adv Exp Med Biol 2013;790:128-149.

3. Overbaugh J, Miller AD, Eiden MV. Receptors and entry cofactors for retroviruses include single and multiple transmembrane-spanning proteins as well as newly described glycophosphatidylinositolanchored and secreted proteins. Microbiol Mol Biol Rev 2001;65:371-389,

4. Coffin JM, Hughes SH, Varmus HE. Retroviruses. Cold Spring Harbor (NY): Cold Spring Harbor Laboratory Press; 1997.

5. Lesbats P, Engelman AN, Cherepanov P. Retroviral DNA Integration. Chem Rev 2016;116:12730-12757.

6. Pornillos 0, Ganser-Pornillos BK. Maturation of retroviruses. Curr Opin Virol 2019:36:47-55

7. Maeda N, Fan H, Yoshikai Y. Oncogenesis by retroviruses: old and new paradigms. Rev Med Virol 2008:18:387-405.

8. Krupovic M, Blomberg J, Coffin JM, Dasgupta I, Fan H, et al. Ortervirales: new virus order unifying five families of reverse-transcribing viruses. J Virol 2018;92:e00515-18 